AdVANCES IN COMBINATORICS, 2020:1, 14 pp.

www.advancesincombinatorics.com

\title{
Tight paths in convex geometric hypergraphs
}

\author{
Zoltán Füredi* $^{*} \quad$ Tao Jiang $^{\dagger} \quad$ Alexandr Kostochka $^{\ddagger} \quad$ Dhruv Mubayi $^{\S}$ \\ Jacques Verstraëte
}

Received 24 June 2018; Published 28 February 2020

\begin{abstract}
In this paper, we prove a theorem on tight paths in convex geometric hypergraphs, which is asymptotically sharp in infinitely many cases. Our geometric theorem is a common generalization of early results of Hopf and Pannwitz [12], Sutherland [19], Kupitz and Perles [16] for convex geometric graphs, as well as the classical Erdős-Gallai Theorem [6] for graphs. As a consequence, we obtain the first substantial improvement on the Turán problem for tight paths in uniform hypergraphs.
\end{abstract}

Key words and phrases: Paths, convex geometric hypergraphs, Turan number

\section{Introduction}

In this paper, we address extremal questions for tight paths in uniform hypergraphs and in convex geometric hypergraphs. For $k \geq 1$ and $r \geq 2$, a tight $k$-path is an $r$-uniform hypergraph (or simply $r$-graph) $P_{k}^{r}=\left\{v_{i} v_{i+1} \ldots v_{i+r-1}: 0 \leq i<k\right\}$. Let ex $\left(n, P_{k}^{r}\right)$ denote the maximum number of edges in an $n$-vertex $r$-graph not containing a tight $k$-path. It appears to be difficult to determine ex $\left(n, P_{k}^{r}\right)$ in general, and even the asymptotics as $n \rightarrow \infty$ are not known. The following is a special case of a conjecture of Kalai [9] on tight trees, generalizing the well-known Erdős-Sós Conjecture [7]:

\footnotetext{
* Supported by grant K116769 from the National Research, Development and Innovation Office NKFIH and by the Simons Foundation Collaboration grant 317487.

$\dagger$ Supported by National Science Foundation award DMS-1400249.

${ }^{\ddagger}$ Supported by NSF grant DMS1600592, by Arnold O. Beckman Award RB20003 of the University of Illinois at UrbanaChampaign and by grants 18-01-00353A and 19-01-00682 of the Russian Foundation for Basic Research.

${ }^{\S}$ Supported by NSF grants DMS-1300138 and DMS-1763317.

I Supported by NSF grants DMS-1556524 and DMS-1800332.
} 
Conjecture 1.1 (Kalai). For $n \geq r \geq 2$ and $k \geq 1$, ex $\left(n, P_{k}^{r}\right) \leq \frac{k-1}{r}\left(\begin{array}{c}n \\ r-1\end{array}\right)$.

A construction based on combinatorial designs shows this conjecture if true is tight - the existence of designs was established by Keevash [13] and also more recently by Glock, Kühn, Lo and Osthus [10]. It is straightforward to see that any $n$-vertex $r$-graph $H$ that does not contain a tight $k$-path has at most $(k-1)\left(\begin{array}{c}n \\ r-1\end{array}\right)$ edges. Patkós [18] gave an improvement over this bound in the case $k<3 r / 4$. In the special case $k=4$ and $r=3$, it is shown in [8] that $\operatorname{ex}\left(n, P_{4}^{3}\right)=\left(\begin{array}{l}n \\ 2\end{array}\right)$ for all $n \geq 5$. In this paper, we give the first non-trivial upper bound on $\operatorname{ex}\left(n, P_{k}^{r}\right)$ valid for all $k$ and $r$ :

Theorem 1.2. For $n \geq 1, r \geq 2$, and $k \geq 1$,

$$
\operatorname{ex}\left(n, P_{k}^{r}\right) \leq \begin{cases}\frac{k-1}{2}\left(\begin{array}{c}
n \\
r-1
\end{array}\right) & \text { if } r \text { is even } \\
\frac{1}{2}\left(k+\left\lfloor\frac{k-1}{r}\right\rfloor\right)\left(\begin{array}{c}
n \\
r-1
\end{array}\right) & \text { if } r \text { is odd }\end{cases}
$$

The case $r=2$ of this result is the well-known Erdôs-Gallai Theorem [6] on paths in graphs. We prove Theorem 1.2 by introducing a novel method for extremal problems for paths in convex geometric hypergraphs.

Convex geometric hypergraphs. A convex geometric hypergraph (or cgh for short) is an $r$-graph whose vertex set is a set $\Omega_{n}$ of $n$ vertices in strictly convex position in the plane, and whose edges are viewed as convex $r$-gons with vertices from $\Omega_{n}$. Given an $r$-uniform $\operatorname{cgh} F$, let ex $(n, F)$ denote the maximum number of edges in an $n$-vertex $r$-uniform cgh that does not contain $F$. Extremal problems for convex geometric graphs (or cggs for short) have been studied extensively, going back to theorems in the 1930's on disjoint line segments in the plane. We refer the reader to the papers of Braß, Károlyi and Valtr [3], Capoyleas and Pach [5] and the references therein for many related extremal problems on convex geometric graphs and to Aronov, Dujmovič, Morin, Ooms and da Silveira [1], Braß [2], Brass, Rote and Swanepoel [4], and Pach and Pinchasi [17] for problems in convex geometric hypergraphs, and their connections to important problems in discrete geometry, as well as the triangle-removal problem (see Aronov, Dujmovič, Morin, Ooms and da Silveira [1] and Gowers and Long [11]).

Concerning results on convex geometric graphs, let $\mathrm{M}_{k}$ denote the cgg consisting of $k$ pairwise disjoint line segments. Generalizing results of Hopf and Pannwitz [12] and Sutherland [19], Kupitz [15] and Kupitz and Perles [16] showed that for $n \geq k \geq 2$,

$$
\operatorname{ex}_{\circlearrowright}\left(n, \mathrm{M}_{k}\right) \leq(k-1) n .
$$

Perles proved the following even stronger theorem. Define a $k$-zigzag $\mathrm{P}_{k}$ to be a $k$-path $v_{0} v_{1} \ldots v_{k}$ with vertices in $\Omega_{n}$ such that in a fixed cyclic ordering of $\Omega_{n}$, the vertices appear in the order $v_{0}, v_{2}, v_{4}, \ldots, v_{5}, v_{3}, v_{1}$, $v_{0}$ (see the left picture in Figure 1).

Theorem 1.3 (Perles). For $n, k \geq 1$, $\operatorname{ex}_{\circlearrowright}\left(n, \mathrm{P}_{k}\right) \leq(k-1) n / 2$.

The bound in Theorem 1.3 is tight when $k$ divides $n$ since any disjoint union of cliques of order $k$ does not contain any path with $k$ edges. In particular, since $\mathrm{P}_{2 k-1}$ contains $\mathrm{M}_{k}$, Theorem 1.3 implies $\operatorname{ex}_{\circlearrowright}\left(n, \mathrm{M}_{k}\right) \leq \operatorname{ex}_{\circlearrowright}\left(n, \mathrm{P}_{2 k-1}\right) \leq(k-1) n$. It appears to be challenging to determine for all $k$ and $r$ the exact value of the extremal function or the extremal cghs without $k$-zigzag (see Keller and Perles [14] for a discussion of extremal constructions in the case $r=2$ ). 


\section{TIGHT PATHS IN HYPERGRAPHS}

In this paper, we generalize Theorem 1.3 to convex geometric hypergraphs, and use its proof technique to prove Theorem 1.2. We let $\prec$ denote a fixed cyclic ordering of the vertices of $\Omega_{n}$, and let $[u, v]=\left\{w \in \Omega_{n}: u \prec w \prec v\right\}$ denote a segment of $\Omega_{n}$. If $I_{1}, I_{2}, \ldots \subset \Omega_{n}$, then we write $I_{1} \prec I_{2} \prec \cdots$ if all vertices of $I_{j}$ are followed in the ordering $\prec$ by all vertices of $I_{j+1}$ for $j \geq 1$. We use the following definition of a path in a convex geometric hypergraph:

Definition 1.4 (Zigzag paths). For $k \geq 1$ and even $r \geq 2$, a tight $k$-path $v_{0} v_{1} \ldots v_{k+r-2}$ with vertices in $\Omega_{n}$ is a $k$-zigzag, denoted $\mathrm{P}_{k}^{r}$, if there exist disjoint segments $I_{0} \prec I_{1} \prec \cdots \prec I_{r-1}$ of $\Omega_{n}$ such that $\left\{v_{i}: i \equiv j(\bmod r)\right\} \subseteq I_{j}$ for $0 \leq j<r$ and

(i) $\quad$ if $j$ is even, then $v_{j} \prec v_{j+r} \prec v_{j+2 r} \prec \cdots$.

(ii) if $j$ is odd, then $v_{j} \succ v_{j+r} \succ v_{j+2 r} \succ \cdots$.

In words, the vertices of the zigzag with subscripts congruent to $j(\bmod r)$ appear in increasing order of subscripts if $j$ is even, followed by the vertices with subscripts congruent to $j+1(\bmod r)$ in decreasing order of subscripts with respect to the cyclic ordering $\prec$. In the case of graphs, a $k$-zigzag is simply $\mathrm{P}_{k}^{2}=\mathrm{P}_{k}$ from Theorem 1.3. We give examples of zigzag paths $\mathrm{P}_{6}^{2}$ and $\mathrm{P}_{5}^{4}$ in Figure 1 below (the last edge of each path is indicated in bold).
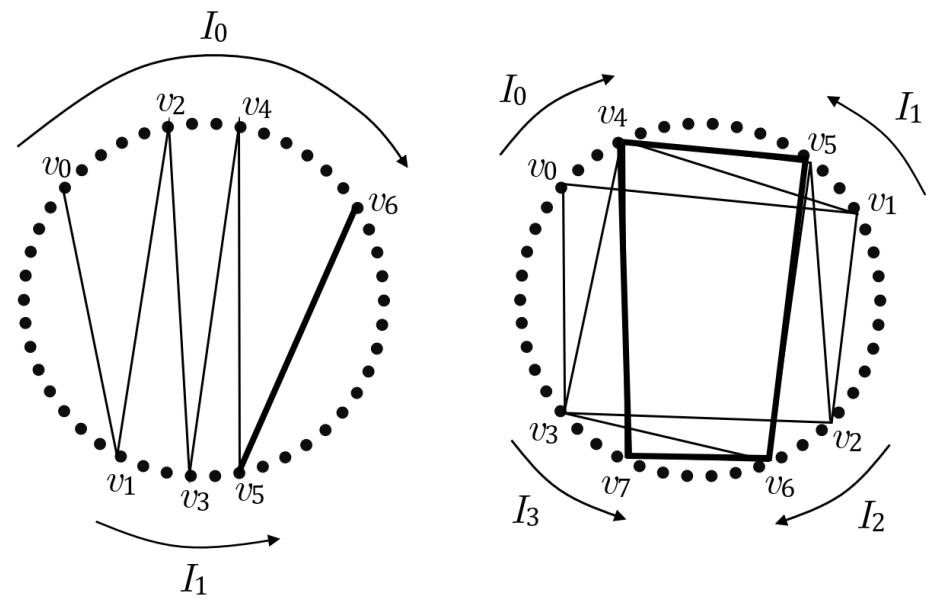

Figure 1: Zigzag paths

The following result generalizes Theorem 1.3 to $r$-uniform cghs when $r$ is even:

Theorem 1.5. Let $n, k \geq 1$, and let $r \geq 2$ be even. Then

$$
\operatorname{ex}_{\circlearrowright}\left(n, \mathrm{P}_{k}^{r}\right) \leq \frac{(k-1)(r-1)}{r}\left(\begin{array}{c}
n \\
r-1
\end{array}\right) .
$$

This theorem is asymptotically sharp in infinitely many cases, and is a common generalization Theorem 1.3 and the Erdős-Gallai Theorem [6]. The proof of Theorem 1.5 is also the basis for our proof of Theorem 1.2. 
Organization. This paper is organized as follows. In Section 2, we give a method for extending a $k$-zigzag in an $r$-uniform cgh to a $(k+1)$-zigzag. This is used in the short proof of Theorem 1.5 in Section 3. In Section 4, we give constructions of dense cghs without $k$-zigzags, and in Section 5, we prove Theorem 1.2 using the proof technique of Theorem 1.5.

Notation. We let $\Omega_{n}$ denote a generic set of $n$ points in strictly convex position in the plane, and let $\prec$ denote a cyclic ordering of $\Omega_{n}$. For $u, v \in \Omega_{n}$, we write $[u, v]=\{w: u \prec w \prec v\}$; this is the set of vertices in the segment of $\Omega_{n}$ from $u$ to $v$ (including $u$ and $v$ ) in the ordering $\prec$. For $u, v \in \Omega_{n}$, let $\ell(u, v)=\min \{|[u, v]|-1,|[v, u]|-1\}$. In other words, $\ell(u, v)$ is the number of sides in a shortest segment of $\Omega_{n}$ between $u$ and $v$. Throughout this paper, cghs have vertex set in $\Omega_{n}$ with cyclic ordering $\prec$. For an $r$-uniform cgh $F$, let ex ${ }_{\circlearrowright}(n, F)$ denote the maximum number of edges in an $r$-uniform cgh on $\Omega_{n}$ that does not contain an ordered substructure isomorphic to $F$. We write $V(H)$ for the vertex set of a hypergraph $H$, and represent the edges as unordered lists of vertices. We identify a hypergraph $H$ with its edge-set, denoting by $|H|$ the number of edges in $H$. For $v \in V(H)$, the neighborhood of $v$ is $N(v)=\bigcup_{v \in e \in H}(e \backslash\{v\})$. Let $\partial H$ denote the shadow of an $r$-graph $H$, namely $\{e \backslash\{x\}: x \in e \in H\}$.

\section{Extending zigzags}

\subsection{Extending zigzags in graphs}

We start with a short proof of Theorem 1.3 for zigzags of odd length, along the lines of Perles' proof, which gives an idea of the proof of Theorem 1.5.

Proposition 2.1. Let $k \geq 0$. If $G$ is an n-vertex cgg with no $(2 k+1)$-zigzag, then $|G| \leq k n$.

Proof. Proceed by induction on $k$; for $k=0$, the statement is clear. Suppose $k \geq 1$ and $G$ is an $n$-vertex $\operatorname{cgg}$ with no $(2 k+1)$-zigzag. For $v \in V(G)$, let $f(v)$ be the first vertex of $N(v)$ after $v$ in the ordering $\prec$. Let $E=\{v f(v): v \in V(G)\}$. If $v_{0} v_{1} \ldots v_{2 k-1}$ is a $(2 k-1)$-zigzag in $F=G \backslash E$, then $f\left(v_{0}\right) v_{0} \ldots v_{2 k-1} f\left(v_{2 k-1}\right)$ is a $(2 k+1)$-zigzag in $G$. So $F$ has no $(2 k-1)$-zigzag, and $|F| \leq(k-1) n$ by induction. Since $|E| \leq n$, $|G|=|F|+|E| \leq k n$.

A key point is that a zigzag $v_{0} v_{1} \ldots v_{k}$ can be extended to a $(k+1)$-zigzag $v_{0} v_{1} \ldots v_{k} v$ if $v$ is adjacent to $v_{k}$ and $v \in\left[v_{k}, v_{k-1}\right]$ if $k$ is even, whereas $v \in\left[v_{k-1}, v_{k}\right]$ if $k$ is odd (the reader may find it helpful to refer to Figure 1). In the next section, we generalize these ideas to uniform cghs.

\subsection{Extending zigzags in hypergraphs}

Fixing an even $r \geq 2$, we write $v_{k}$ as shorthand for $\left(v_{k-1}, v_{k}, \ldots, v_{k+r-2}\right)$. We use this as notation for the ordering of the last edge of a $k$-zigzag:

Definition 2.2. The end of a $k$-zigzag $v_{0} v_{1} \ldots v_{k+r-2}$ is $v_{k}=\left(v_{k-1}, v_{k}, \ldots, v_{k+r-2}\right)$. Let $I\left(v_{k}\right)=\left[v_{k-1}, v_{k}\right]$ if $k$ is odd and $I\left(v_{k}\right)=\left[v_{k+r-2}, v_{k-1}\right]$ if $k$ is even, and

$$
X\left(v_{k}\right)=\left\{v \in I\left(v_{k}\right): v v_{k} v_{k+1} \ldots v_{k+r-2} \in H\right\}
$$




\section{TIGHT PATHS IN HYPERGRAPHS}

Referring to Figure 1, in the picture on the left $X\left(v_{6}\right)$ is the set of $v$ in the segment from $v_{6}$ to $v_{5}$ clockwise such that $v_{6} v$ is an edge. In the picture on the right, $X\left(v_{5}\right)$ is the set of $v$ in the segment from $v_{4}$ to $v_{5}$ clockwise such that $v_{5} v_{6} v_{7} v$ is an edge. In the next proposition, we see that any vertex in $X\left(v_{k}\right)$ can be used to "extend" a $k$-zigzag ending in $v_{k}$ to a $(k+1)$-zigzag:

Proposition 2.3. Let $v_{k} \in V(H)^{r}$ be the end of a k-zigzag $\mathrm{P}$ in $H$. Then for any $v_{k+r-1} \in X(v), \mathrm{P} \cup$ $\left\{v_{k} v_{k+1} \ldots v_{k+r-1}\right\}$ is a $(k+1)$-zigzag ending in $v_{k+1}$.

Proof. Let $\mathrm{P}=v_{0} v_{1} \ldots v_{k+r-2}$ and let $I_{0} \prec I_{1} \prec \cdots \prec I_{r-1}$ be the segments in Definition 1.4. Let $v_{k-1} \in I_{j}$, so $j \equiv k-1(\bmod r)$. If $k$ is odd, then $j$ is even, and the vertices of $I_{j} \cup I_{j+1}$ appear in the order $v_{j} \prec \cdots \prec$ $v_{k-1} \prec v_{k} \prec \cdots \prec v_{j+1}$ by Definition 1.4(i). Then for any $v_{k+r-1} \in X\left(v_{k}\right), e=v_{k} v_{k+1} \ldots v_{k+r-2} v_{k+r-1} \in H$ and adding $e$ to $\mathrm{P}$ and $v_{k+r-1}$ to $I_{j}$ before $v_{k-1}$ in the clockwise orientation, we obtain a $(k+1)$-zigzag. Similarly, if $k$ is even, then $j$ is odd so the vertices of $I_{j-1} \cup I_{j}$ appear in the order $v_{j-1} \prec \cdots \prec v_{k+r-2} \prec$ $v_{k-1} \prec \cdots \prec v_{j}$ by Definition 1.4(ii), and we add $e$ to $\mathrm{P}$ and $v_{k+r-1}$ after $v_{k-1}$ in $I_{j}$ in the clockwise orientation.

Definition 2.4. Let $S_{k}(H)$ be the set of ends $v_{k} \in V(H)^{r}$ of $k$-zigzags in $H$, and

$$
T_{k}(H)=\left\{v_{k} \in S_{k}(H): X\left(v_{k}\right)=\emptyset\right\} .
$$

Informally, $T_{k}(H)$ is the set of ends of $k$-zigzags which cannot be "extended" to $(k+1)$-zigzags. The two key propositions for the proof of Theorem 1.5 are as follows.

Proposition 2.5. For $v_{k} \in S_{k}(H) \backslash T_{k}(H)$, let $v_{k+r-1} \in X\left(v_{k}\right)$ be as close as possible to $v_{k-1}$ in the segment $I\left(v_{k}\right)$. Then $f\left(v_{k}\right)=v_{k+1}$ is an injection from $S_{k}(H) \backslash T_{k}(H)$ to $S_{k+1}(H)$. In particular,

$$
\left|S_{k+1}(H)\right| \geq\left|S_{k}(H) \backslash T_{k}(H)\right| \text {. }
$$

Proof. By Proposition 2.3, $f\left(v_{k}\right) \in S_{k+1}(H)$. Furthermore, $f\left(v_{k}\right)=f\left(w_{k}\right)$ implies $v_{k+1}=w_{k+1}$, which gives $v_{i}=w_{i}$ for $k \leq i \leq k+r-1$. If $v_{k-1} \neq w_{k-1}$, then either $w_{k-1}$ is closer to $v_{k-1}$ than $w_{k+r-1}$ in $I\left(v_{k}\right)$, or $v_{k-1}$ is closer to $w_{k-1}$ than $v_{k+r-1}$ in $I\left(v_{k}\right)$. These contradictions imply $v_{k-1}=w_{k-1}$, and so $v_{k}=w_{k}$ and $f$ is an injection.

Proposition 2.6. For $v_{k} \in T_{k}(H)$, the map $g\left(v_{k}\right)=\left(v_{k}, v_{k+1}, \ldots, v_{k+r-2}\right)$ is an injection from $T_{k}(H)$ to cyclically ordered elements of $\partial H$. In particular,

$$
\left|T_{k}(H)\right| \leq(r-1)|\partial H|
$$

Proof. If $g\left(v_{k}\right)=g\left(w_{k}\right)$, then $w_{i}=v_{i}$ for $k \leq i \leq k+r-2$. Suppose $v_{k-1} \neq w_{k-1}$. Then either $v_{k+r-2} \prec$ $w_{k-1} \prec v_{k-1}$, and $v_{k-1} \in X\left(w_{k}\right)$, or $v_{k-1} \prec w_{k-1} \prec v_{k}$, and $w_{k-1} \in X\left(v_{k}\right)$. In either case, $v_{k} \notin T_{k}$ or $w_{k} \notin T_{k}$, a contradiction. So $v_{k-1}=w_{k-1}$, which implies $v_{k}=w_{k}$. 


\section{Proof of Theorem 1.5 on zigzags}

The following theorem implies Theorem 1.5, since if $H$ is an $n$-vertex $r$-uniform cgh not containing a $k$-zigzag, then $S_{k}(H)=\emptyset$, and we always have $|\partial H| \leq\left(\begin{array}{c}n \\ r-1\end{array}\right)$.

Theorem 3.1. Let $k \geq 1$ and let $r \geq 2$ be even. Then for any $r$-uniform cgh $H$,

$$
\left|S_{k}(H)\right| \geq r|H|-(r-1)(k-1)|\partial H| .
$$

Proof. We prove (3.1) by induction on $k$. Let $k=1$ and $e \in H$. By Definition 1.4(i), there are $r$ possible orderings of the vertices of $e$ giving a 1-zigzag: having chosen the first vertex, the ordering of the remaining vertices of $e$ is determined. Therefore $\left|S_{1}(H)\right| \geq r|H|$. For the induction step, suppose $k \geq 1$ and (3.1) holds. By (2.1) and (2.2),

$$
\begin{aligned}
\left|S_{k+1}(H)\right| & \geq\left|S_{k}(H) \backslash T_{k}(H)\right| \geq r|H|-(r-1)(k-1)|\partial H|-\left|T_{k}(H)\right| \\
& \geq r|H|-(r-1) k|\partial H| .
\end{aligned}
$$

This proves (3.1).

\section{Stack-free constructions}

Let $k \geq 1$ and let $r \geq 2$ be even. A $k$-stack, denoted $\mathrm{M}_{k}^{r}$, consists of edges $\left\{v_{i r}, v_{i r+1}, \ldots, v_{i r+r-1}: 0 \leq i<k\right\}$ where $v_{0} v_{1} \ldots v_{(k-1) r-1}$ is an $r$-uniform zigzag path; in other words we pick every $r$ th edge from a zigzag path $\mathrm{P}_{(k-1) r+1}^{r}$. An example for $r=4$ and $k=7$ is shown below, where the extreme points on the perimeter form $\Omega_{28}$.

There is a simple construction of an $r$-uniform cgh with no $k$-stack when $k$ is odd with $(k-1)(r-$ 1) $\left(\begin{array}{c}n \\ r-1\end{array}\right)+O\left(n^{r-2}\right)$ edges. If $k \geq 3$ is odd, let $H$ be the cgh consisting of $r$-sets $e$ from $\Omega_{n}$ such that $\ell(u, v) \leq k-1$ for some $u, v \in e$. It is straightforward to see that $|H|=(r-1)(k-1)\left(\begin{array}{c}n \\ r-1\end{array}\right)+O\left(n^{r-2}\right)$, and $H$ contains no $k$-stack since the "middle" edge $e$ in the stack - drawn in bold in Figure 2 - has $\ell(u, v) \geq k$ for all $u, v \in e$.

In this section, we extend this construction to all values of $k$, thereby proving the following theorem, which may be of independent interest. In particular, this construction does not contain $\mathrm{P}_{(k-1) r+1}^{r}$, and shows Theorem 1.5 is asymptotically tight for zigzags of length $1(\bmod r)$.

Theorem 4.1. Let $k \geq 1$ and $r \geq 2$ be even. Then

$$
\operatorname{ex}_{\circlearrowright}\left(n, \mathrm{M}_{k}^{r}\right)=(k-1)(r-1)\left(\begin{array}{c}
n \\
r-1
\end{array}\right)+O\left(n^{r-2}\right) .
$$

Proof. We have $\operatorname{ex}_{\circlearrowright}\left(n, \mathrm{M}_{k}^{r}\right) \leq(k-1)(r-1)\left(\begin{array}{c}n \\ r-1\end{array}\right)$ from Theorem 1.5. The main part of the proof is the construction of an $r$-uniform cgh with $(k-1)(r-1)\left(\begin{array}{c}n \\ r-1\end{array}\right)+O\left(n^{r-2}\right)$ edges that does not contain a $k$-stack. 


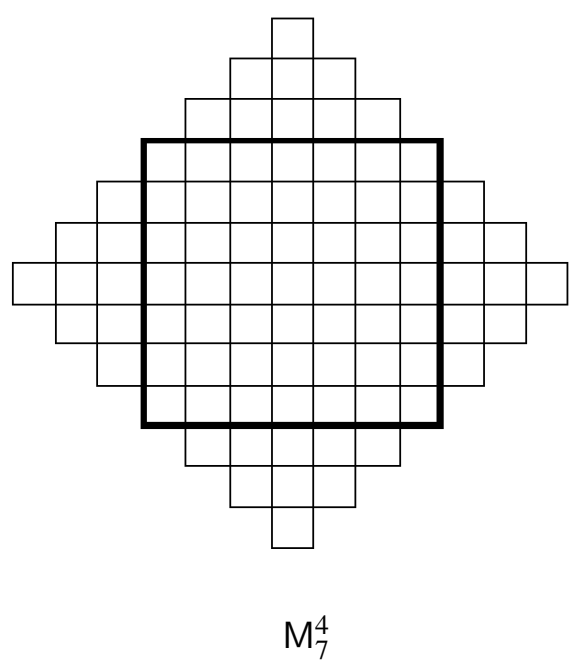

Figure 2: Stack

It will be convenient to let $\Omega_{n}=\{0,1,2, \ldots, n-1\}$ in cyclic order, and view our edges as ordered $r$-tuples $\left(v_{0}, v_{1}, \ldots, v_{r-1}\right)$ where $0 \leq v_{0}<v_{1}<\ldots<v_{r-1} \leq n-1$.

Our construction $H=H(n, r, k)$ has the form $H=\bigcup_{j=0}^{k-1} H_{j}$, where

(i) $H_{0}=\left\{\left(v_{0}, v_{1}, v_{2}, \ldots, v_{r-1}\right): v_{0}=0\right\}$,

(ii) $H_{j}=\bigcup_{h=0}^{r-1}\left\{\left(v_{0}, v_{1}, \ldots, v_{r-1}\right) \notin H_{0}: \ell\left(v_{h}, v_{h+1}\right)=j\right\}$ for $1 \leq j \leq k-2$,

(iii) $H_{k-1}=\bigcup_{h=1}^{r / 2-1}\left\{\left(v_{0}, v_{1}, \ldots, v_{r-1}\right) \notin H_{0}: \ell\left(v_{2 h-1}, v_{2 h}\right) \in\{k-1, k\}\right\}$.

Claim 1. $|H|=(k-1)(r-1)\left(\begin{array}{c}n \\ r-1\end{array}\right)+O\left(n^{r-2}\right)$.

Proof. By definition, $\left|H_{0}\right|=\left(\begin{array}{c}n-1 \\ r-1\end{array}\right)$, and $H_{0} \cap \bigcup_{j=1}^{k-1} H_{j}=\emptyset$. For any $j: 1 \leq j \leq k-2$, as $n \rightarrow \infty$,

$$
\left|H_{j}\right|=(n-1)\left(\begin{array}{c}
n-j-1 \\
r-2
\end{array}\right)+O\left(n^{r-2}\right)=(r-1)\left(\begin{array}{c}
n \\
r-1
\end{array}\right)+O\left(n^{r-2}\right)
$$

and also

$$
\left|H_{k-1}\right|=2(r / 2-1)\left(\begin{array}{c}
n-1 \\
r-1
\end{array}\right)+O\left(n^{r-2}\right)=(r-2)\left(\begin{array}{c}
n \\
r-1
\end{array}\right)+O\left(n^{r-2}\right) .
$$

If $1 \leq i<j \leq k-1,\left|H_{i} \cap H_{j}\right|=O\left(n^{r-2}\right)$. By inclusion-exclusion,

$$
|H| \geq\left|H_{0}\right|+\sum_{j=1}^{k-1}\left|H_{j}\right|-\sum_{i<j}\left|H_{i} \cap H_{j}\right|=(k-1)(r-1)\left(\begin{array}{c}
n \\
r-1
\end{array}\right)+O\left(n^{r-2}\right) .
$$

This proves the claim.

Claim 2. $\mathrm{M}_{k}^{r} \not \subset H$. 
Proof. Suppose $H$ contains a $k$-stack. The key is to consider the "middle" two edges of the stack, say $e$ and $f$. Then the vertex 0 is in at most one of $e$ and $f$. If $v_{0}$ is the first vertex of $e$ and $w_{0}$ is the first vertex of $f$ after 0 in the clockwise direction, then without loss of generality we may assume $v_{0}<w_{0}$. Now consider the pairs $w_{1} w_{2}, w_{3} w_{4}$ up to $w_{r-1} w_{r}$ which are in $f$. We claim all of these pairs have length at least $k+1$, contradicting the definition of $H$, since $f$ would then not be a member of $H$. To see the claim, fix $h: 1 \leq h<r / 2$. Notice that there are $k / 2$ edges of the stack (excluding $f$ ) which contain a pair of vertices in the segment $\left[w_{2 h-1}, w_{2 h}\right]$, and these pairs are vertex disjoint. However, then $\ell\left(w_{2 h-1}, w_{2 h}\right) \geq 2(k / 2+1)-1=k+1$, and this holds for $1 \leq h<r / 2$.

\section{Proof of Theorem 1.2 on tight paths}

Proof for $r$ even. Let $H$ be an $n$-vertex $r$-graph with no tight $k$-path, where $r$ is even. We aim to prove the following, which gives Theorem 1.2 for $r$ even:

$$
|H| \leq \frac{k-1}{2}|\partial H| .
$$

We follow the approach used to prove Theorem 1.5 on a carefully chosen subgraph $G$ of $H$. This subgraph is defined via a random partition of $V(H):$ let $s=r / 2$ and let $\chi: V(G) \rightarrow\{0,1, \ldots, s-1\}$ be a random $s$-coloring of the vertices of $H$ such that $\mathrm{P}(\chi(v)=i)=1 / s$ for $0 \leq i \leq s-1$ and each vertex $v \in V(H)$, and such that vertices are colored independently. Let $B_{i}=\{v \in V(H): \chi(v)=i\}$, and define the following (random) subgraph of $H$ :

$$
G=\left\{e \in H:\left|e \cap B_{i}\right|=2 \text { for } 0 \leq i \leq s-1\right\} .
$$

In other words, each edge of $G$ has two vertices in each of the sets $B_{i}$. For $0 \leq i \leq s-1$, let

$$
\partial_{i} G=\left\{e \in \partial G:\left|e \cap B_{i}\right|=1\right\} \subset\left\{e \in \partial H:\left|e \cap B_{i}\right|=1,\left|e \cap B_{j}\right|=2 \text { for } j \neq i\right\} .
$$

Then we have the following expected values:

$$
\mathrm{E}(|G|)=\frac{r !}{2^{s} s^{r}}|H| \quad \text { and } \quad \mathrm{E}\left(\left|\partial_{i} G\right|\right) \leq \frac{(r-1) !}{2^{s-1} s^{r-1}}|\partial H| .
$$

The next step is to introduce some geometric structure on $G$. Let $\prec$ denote a cyclic ordering of the vertices of each of $B_{0}, B_{1}, \ldots, B_{s-1}$.

Definition 5.1 (Good paths). We call a tight path $v_{0} v_{1} \ldots v_{k+r-2}$ in $G$ good if

(i) for $0 \leq j<k+r-2, v_{j}, v_{j+1} \in B_{i}$ whenever $j \equiv 2 i(\bmod r)$.

(ii) the cyclic order in $B_{i}$ is always $v_{j} \prec v_{j+r} \prec v_{j+2 r} \prec \ldots \prec v_{j+1+2 r} \prec v_{j+1+r} \prec v_{j+1}$.

An $r$-uniform good path with $k$ edges is shown in Figure 3, for $r=6$ and $k=4$.

We now follow the ideas in Section 2. By Definition 5.1(i), $v_{j} \in B_{i}$ if and only if $i=h(j)=$ $\lfloor j / 2\rfloor(\bmod s)$. Let $i=h(k-1)$, so that $v_{k-1} \in B_{i}$. We write $[u, v]=\left\{w \in B_{i}: u \prec w \prec v\right\}$. Define $I\left(v_{k}\right)=\left[v_{k-1}, v_{k}\right] \subseteq B_{i}$ if $k$ is odd and $I\left(v_{k}\right)=\left[v_{k+r-2}, v_{k-1}\right] \subseteq B_{i}$ if $k$ is even, and

$$
X\left(v_{k}\right)=\left\{v \in I\left(v_{k}\right): v v_{k} v_{k+1} \ldots v_{k+r-2} \in H\right\}
$$




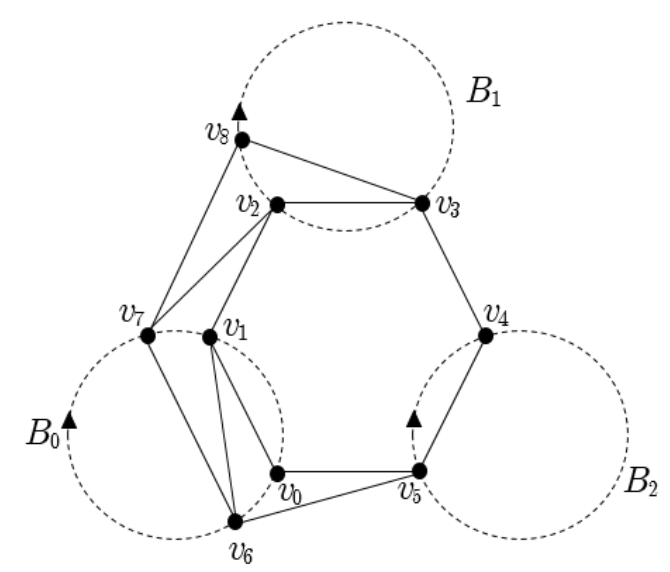

Figure 3: Good paths

Note that the definition of $X\left(v_{k}\right)$ is identical to that in Section 2 but with respect to the ordering $\prec$ of $B_{i}$, where $i=h(k-1)$, and in particular, $I\left(v_{k}\right), X\left(v_{k}\right) \subseteq B_{i}$. In Figure 3, $X\left(v_{4}\right)$ consists of all verteices $v \in B_{1}$ clockwise from $v_{8}$ to $v_{3}$ such that $v_{4} v_{5} v_{6} v_{7} v_{8} v \in G$. Let $S_{k}(G)$ be the set of the ends of good $k$-paths in $G$, and let $T_{k}(G)=\left\{v_{k} \in S_{k}(H): X\left(v_{k}\right)=\emptyset\right\}$.

Claim 1. For $k \geq 1$, if $i=h(k-1)$, then

$$
\left|T_{k}(G)\right| \leq 2^{s-1}\left|\partial_{i} G\right|
$$

Proof. If $v_{k} \in S_{k}(G)$, then $v_{k-1} \in B_{i}$ since $i=h(k-1)$. For $v_{k} \in T_{k}(G)$, define $g\left(v_{k}\right)=\left(v_{k}, v_{k+1}, \ldots\right.$, $\left.v_{k+r-2}\right)$. Then $v_{k} v_{k+1} \ldots v_{k+r-2} \in \partial_{i} G$ and $\left(v_{k}, v_{k+1}, \ldots, v_{k+r-2}\right)$ is uniquely determined by specifying the order of the pairs $\left\{v_{k}, v_{k+1}, \ldots, v_{k+r-2}\right\} \cap B_{j}$ for each $j \neq i$. Therefore $g\left(v_{k}\right)$ injectively maps elements of $T_{k}(G)$ to ordered elements of $\partial_{i} G$, where each element of $\partial_{i} G$ is ordered in $2^{s-1}$ ways. We conclude $\left|T_{k}(G)\right| \leq 2^{s-1}\left|\partial_{i} G\right|$.

Claim 2. For $k \geq 1$,

$$
\left|S_{k}(G)\right| \geq 2^{s}|G|-2^{s-1} \sum_{i=0}^{k-2}\left|\partial_{h(i)} G\right| .
$$

Proof. For $k=1$, we observe for $e \in G$, there are two ways to label the pair $e \cap B_{i}$ for each $i \in[s]$, and therefore $\left|S_{1}(G)\right| \geq 2^{s}|G|$. Suppose (5.4) holds for some $k \geq 1$. Then we copy the proofs of Propositions 2.3 and 2.5 to obtain $\left|S_{k+1}(G)\right| \geq\left|S_{k}(G) \backslash T_{k}(G)\right|$. By the induction hypothesis (5.4) and Claim 1,

$$
\begin{aligned}
\left|S_{k+1}(G)\right| \geq\left|S_{k}(G) \backslash T_{k}(G)\right| & \geq 2^{s}|G|-2^{s-1} \sum_{i=0}^{k-2}\left|\partial_{h(i)} G\right|-\left|T_{k}(G)\right| \\
& \geq 2^{s}|G|-2^{s-1} \sum_{i=0}^{k-1}\left|\partial_{h(i)} G\right| .
\end{aligned}
$$


This completes the induction step and proves (5.4).

Proof of (5.1). Finally we prove (5.1). Taking expectations on both sides of (5.4), and using (5.2) and the linearity of expectation:

$$
\mathrm{E}\left(\left|S_{k}(G)\right|\right) \geq 2^{s} \mathrm{E}(|G|)-2^{s-1} \sum_{i=0}^{k-2} \mathrm{E}\left(\left|\partial_{h(i)} G\right|\right) \geq \frac{r !}{s^{r}}|H|-\frac{(r-1) !(k-1)}{s^{r-1}}|\partial H| .
$$

Since $G \subseteq H$ has no tight $k$-path, $S_{k}(G)=\emptyset$. Using this in (5.5), we obtain (5.1).

Proof for $r$ odd. Let $H$ be an $n$-vertex $r$-graph containing no tight $k$-path. We aim to show

$$
|H| \leq \frac{1}{2}\left(k+\left\lfloor\frac{k-1}{r}\right\rfloor\right)|\partial H| .
$$

To prove (5.6), we reduce the case $r$ is odd to the case $r$ is even, and apply (5.1) from the last proof. Form the $(r+1)$-graph $H^{+}$by adding a set $X$ of vertices to $V(H)$, and let $H^{+}=\{\{x\} \cup e: x \in X, e \in H\}$. It is convenient to let $\phi(\ell)=\lceil(\ell+r) /(r+1)\rceil$ for $\ell \geq 1$.

It is straightforward to check that if $P=v_{0} v_{1} \ldots v_{\ell+r-1}$ is a tight $\ell$-path in $H^{+}$, then $|V(P) \cap X| \leq \phi(\ell)$. In addition, the sequence of vertices $v_{i} \in V(P) \backslash X$ in increasing order of subscripts forms a tight path in $H$ of length at least $\ell+1-\phi(\ell)$. Setting $\ell=k+\lfloor(k-1) / r\rfloor+1$, we have $\ell+1-\phi(\ell)=k$, and therefore $H^{+}$has no tight $\ell$-path. By (5.1) applied to $H^{+}$,

$$
\left|H^{+}\right| \leq \frac{\ell-1}{2}\left|\partial H^{+}\right| .
$$

Since $\left|H^{+}\right|=|X||H|$ and $\left|\partial H^{+}\right|=|X||\partial H|+|H|$, we find

$$
|X||H| \leq \frac{\ell-1}{2}|X||\partial H|+\frac{\ell-1}{2}|H| .
$$

Choosing $|X|>(\ell-1)|H| / 2$ and dividing by $|X|$, we obtain $|H| \leq(\ell-1)|\partial H| / 2$. Since $(\ell-1) / 2=$ $(k+\lfloor(k-1) / r\rfloor) / 2$, this proves (5.6).

\section{Concluding remarks}

- It turns out using Steiner systems with arbitrarily large block sizes $[10,13])$ that for each fixed $k, r \geq 2$, both of the following limits exist:

$$
z(k, r):=\lim _{n \rightarrow \infty} \frac{\operatorname{ex}_{\circlearrowright}\left(n, \mathrm{P}_{k}^{r}\right)}{\left(\begin{array}{c}
n \\
r-1
\end{array}\right)} \quad \text { and } \quad p(k, r):=\lim _{n \rightarrow \infty} \frac{\operatorname{ex}\left(n, P_{k}^{r}\right)}{\left(\begin{array}{c}
n \\
r-1
\end{array}\right)} .
$$

The first limit is determined by Theorem 1.5 and the construction in Section 4 for $k \equiv 1(\bmod r)$, and for $r \geq 4$ the problem is wide open in all remaining cases, even for $k=2$.

- For $k \leq r+1$, an improvement over Theorem 1.2 is possible, slightly improving the results of Patkós [18]: we prove by induction on $r$ that if $r \geq k-1$, the

$$
\operatorname{ex}\left(n, P_{k}^{r}\right) \leq \frac{k^{2}}{2 r}\left(\begin{array}{c}
n \\
r-1
\end{array}\right)
$$




\section{TIGHT PATHS IN HYPERGRAPHS}

If $r=k-1$, this follows from Theorem 1.2. Suppose $r \geq k$ and we have proved the bound for $(r-1)$ graphs. Let $H$ be an $r$-graph with no tight $k$-path and pick a vertex $v \in V(H)$ contained in at least $r|H| / n$ edges of $H$. Consider the link hypergraph $H_{v}=\{e \in \partial H: e \cup\{v\} \in H\}$. Then $H_{v}$ has no tight $k$-path, otherwise adding $v$ to each edge we get a tight $k$-path in $H$. By induction,

$$
\frac{r|H|}{n} \leq\left|H_{v}\right| \leq \frac{k^{2}}{2(r-1)}\left(\begin{array}{c}
n-1 \\
r-2
\end{array}\right) \leq \frac{k^{2}}{2 n}\left(\begin{array}{c}
n \\
r-1
\end{array}\right)
$$

and this implies $|H| \leq \frac{k^{2}}{2 r}\left(\begin{array}{c}n \\ r-1\end{array}\right)$, as required.

- It is possible when $r \geq 3$ is odd to obtain a very slight improvement over Theorem 1.2, namely

$$
\operatorname{ex}\left(n, P_{k}^{r}\right) \leq \frac{1}{r}(\sqrt{a}+\sqrt{b})^{2}\left(\begin{array}{c}
n \\
r-1
\end{array}\right)
$$

where $a=\lfloor(k-1) / r\rfloor$ and $b=(r-1)(k-1-a) / 2$ and $n$ is sufficiently large. For the purpose of comparison, we obtain

$$
p(k, r) \leq k \cdot\left(\frac{1}{2}+\frac{\sqrt{2}-1}{r}+c\right)
$$

where $c=O\left(r^{-2}\right)$. For $r=3$, we find that the upper bound is at most $\frac{1}{9}(3+\sqrt{8}) k \cdot\left(\begin{array}{l}n \\ 2\end{array}\right)$.

- The proof in Section 5 shows that if $s=r / 2, n$ is a multiple of $s$, and $G$ is an $n$-vertex $r$-graph such that $V(G)$ is partitioned into sets $B_{0}, B_{1}, \ldots, B_{s-1}$ with $\left|B_{i}\right|=n / s$ and $\left|e \cap B_{i}\right|=2$ for $0 \leq i<s$ and every edge $e \in G$, then $|G| \leq 2^{s-1}(k-1)(n / r)^{r-1}$, and this is asymptotically tight if $k \equiv 1(\bmod r)$. Indeed, let $B_{0}, B_{1}, \ldots, B_{s-1}$ be disjoint sets of size $n / s$, and let $A_{i} \subset B_{i}$ have size $(k-1) / r$. Then let $G_{i}$ consist of all $r$-sets with one vertex in $A_{i}$, one vertex in $B_{i} \backslash A_{i}$, and two vertices in each $B_{j} \backslash A_{j}$ for $0 \leq j<s, j \neq i$. Let $G=\bigcup_{i=0}^{s-1} G_{i}$. Then $|e \cap f| \leq r-2$ for $e \in G_{i}$ and $f \in G_{j}$ with $i \neq j$, so if $G$ contains a tight $k$-path, then the tight $k$-path is contained in some $G_{i}$. However, $A_{i}$ is a transversal of each $G_{i}$, so $G_{i}$ cannot contain a tight $k$-path. Therefore $G$ has no tight $k$-path, and furthermore

$$
|G|=\sum_{i=0}^{s-1}\left|G_{i}\right|=s \frac{k-1}{r} \frac{n}{s}\left(\begin{array}{c}
n / s \\
2
\end{array}\right)^{s-1}+O\left(n^{r-2}\right)=2^{s-1}(k-1)\left(\frac{n}{r}\right)^{r-1}+O\left(n^{r-2}\right) .
$$

- In forthcoming work, we consider extremal problems for various other analogs of paths and matchings in the setting of convex geometric hypergraphs, having considered only zigzag paths and stacks of even uniformity in this paper.

\section{Acknowledgments}

The last author would like to thank Gil Kalai for stimulating discussions occuring at the Oberwolfach Combinatorics Conference, 2017. This research was partly conducted during AIM SQuaRes (Structured Quartet Research Ensembles) workshops, and we gratefully acknowledge the support of AIM. The authors are also grateful to a referee for carefully reading the paper and providing many valuable comments that improved the presentation. 
Zoltán Füredi, Tao Jiang, Alexandr Kostochka, Dhruv Mubayi and Jacques Verstraëte

\section{References}

[1] B. Aronov, V. Dujmovič, P. Morin, A. Ooms, L. da Silveira, More Turán-type theorems for triangles in convex point sets, Electron. J. Combin. 26 (2019), no. 1, Paper 1.8, 26 pp. 2

[2] P. Braß, Turán-type extremal problems for convex geometric hypergraphs, Contemporary Mathematics, 342, 25-34, 2004. 2

[3] P. Braß, G. Károlyi, P. Valtr, A Turán-type extremal theory of convex geometric graphs, GoodmanPollack Festschrift, Springer 2003, 277-302. 2

[4] P. Braß, G. Rote, K. Swanepoel, Triangles of extremal area or perimeter in a finite planar point set, Discrete Comp. Geom., 26 (1), 51-58, 2001. 2

[5] V. Capoyleas, J. Pach, A Turán-type theorem for chords of a convex polygon, J. Combin. Theory Ser. B, 56, 9-15. 2

[6] P. Erdős and T. Gallai, On maximal paths and circuits of graphs, Acta Math. Acad. Sci. Hungar. 10 (1959), 337-356. 1, 2, 3

[7] P. Erdős, Extremal problems in graph theory M. Fiedler (Ed.), Theory of Graphs and its Applications, Academic Press (1965), pp. 29-36. 1

[8] Z. Füredi, T. Jiang, A. Kostochka, D. Mubayi, J. Verstraëte, Hypergraphs not containing a tight tree with a bounded trunk, SIAM J. Discrete Math. 33 (2019), no. 2, 862-873. 2

[9] P. Frankl, Z. Füredi, Exact solution of some Turán-type problems. J. Comb. Theory Ser. A 45 (2), (1987), 226-262. 1

[10] S. Glock, D. Kühn, A. Lo, D. Osthus, The existence of designs via iterative absorption, Preprint: https://arxiv.org/abs/1611.06827, 63 pages. 2, 10

[11] W. T. Gowers, E. Long, The length of an $s$-increasing sequence of $r$-tuples, Preprint: https://arxiv.org/abs/1609.08688. 2

[12] H. Hopf and E. Pannwitz: Aufgabe Nr. 167, Jahresbericht d. Deutsch. Math. Verein. 43 (1934), 114. 1,2

[13] P. Keevash, The existence of designs II, Preprint: https://arxiv.org/abs/1802.05900, 39 pages. 2, 10

[14] C. Keller, M. Perles, On convex geometric graphs with no $k+1$ pairwise disjoint edges. Graphs Combin. 32 (2016), no. 6, 2497-2514. 2

[15] Y. S. Kupitz, On Pairs of Disjoint Segments in Convex Position in the Plane, Annals Discrete Math., 20 (1984), 203-208. 2

[16] Y. S. Kupitz, M. Perles, Extremal theory for convex matchings in convex geometric graphs, Discrete Comput Geom. 15, (1996), 195-220. 1, 2 
[17] J. Pach, R. Pinchasi, How many unit equilateral triangles can be generated by $n$ points in general position? Amer. Math. Monthly 110 (2003), 100-106. 2

[18] B. Patkós, A note on traces of set families, Moscow Journal of Combinatorics and Number Theory 2 (2012), 47-55. See detailed calculations in: On tight paths of fixed length, http://www.renyi.hu/ patkos/tight-paths-fixed.pdf. Posted on 2 Dec 2017, 3 pp. 2, 10

[19] J. W. Sutherland, Lösung der Aufgabe 167, Jahresbericht Deutsch. Math.-Verein. 45 (1935), 33-35. 1,2

\section{AUTHORS}

Zoltán Füredi

Alfréd Rényi Institute of Mathematics

Hungarian Academy of Sciences

Reáltanoda utca 13-15.

H-1053, Budapest, Hungary.

zfuredi@gmail.com

Tao Jiang

Department of Mathematics

Miami University

Oxford, OH 45056, USA.

jiangt@miamioh.edu

Alexandr Kostochka

University of Illinois at Urbana-Champaign

Urbana, IL 61801

and Sobolev Institute of Mathematics

Novosibirsk 630090, Russia.

kostochk@math.uiuc.edu

Dhruv Mubayi

Department of Mathematics, Statistics

and Computer Science

University of Illinois at Chicago

Chicago, IL 60607.

mubayi@uic.edu 
Zoltán Füredi, Tao Jiang, Alexandr Kostochka, Dhruv Mubayi and JacQues Verstraëte

\author{
Jacques Verstraëte \\ Department of Mathematics \\ University of California at San Diego \\ 9500 Gilman Drive, La Jolla, California 92093-0112, USA. \\ jverstra@math.ucsd.edu.
}

